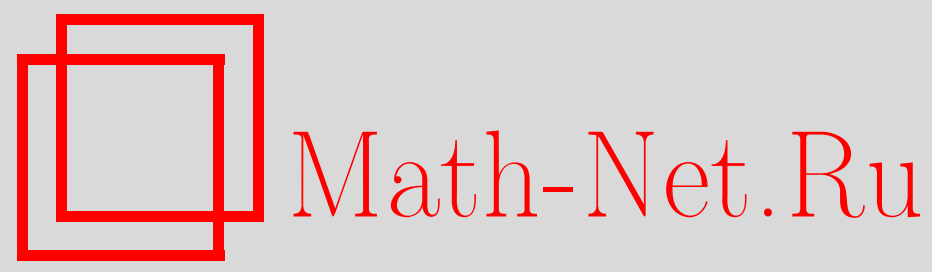

Б. А. Севастьянов, Вычисление предельных вероятностей распределения перманента случайной матрицы в поле $G F(p)$, Дискрет. матем., 2010, том 22, выпуск 3, 3-7

DOI: https://doi.org/10.4213/dm1102

Использование Общероссийского математического портала Math-Net.Ru подразумевает, что вы прочитали и согласны с пользовательским соглашением http://www . mathnet.ru/rus/agreement

Параметры загрузки:

IP: 34.229 .45 .116

26 апреля 2023 г., 18:10:17 


\title{
Вычисление предельных вероятностей распределения перманента случайной матрицы в поле $G F(p)$
}

\author{
(ㄷ) 2010 г. Б. А. Севастьянов
}

Предложен алгоритм вычисления предельных вероятностей

$$
\lim _{n \rightarrow \infty} \mathbf{P}\left\{\operatorname{per}\left(A_{m n}\right)=k\right\}, \quad k=0,1,2, \ldots, p-1, \quad m=1,2,3, \ldots,
$$

перманента случайной матрицы $A_{m n}=\left\|\alpha_{i j}\right\|$ с $m$ строками и $n$ независимыми столбцами в поле $G F(p)$.

Работа выполнена при поддержке Российского фонда фундаментальных исследований, проект 08-01-00078, и программы РАН «Математическая теория управления».

\section{1. Предварительные результаты}

Перманент матрицы $A_{m n}=\left\|\alpha_{i j}\right\|$ с $m$ строками и $n$ столбцами, $n \geqslant m$, определяется формулой

$$
\operatorname{per}\left(A_{m n}\right)=\sum_{\left(j_{1}, j_{2}, \ldots, j_{m}\right)} \alpha_{1 j_{1}} \alpha_{2 j_{2}} \ldots \alpha_{m j_{m}},
$$

где сумма берется по всем наборам попарно различных индексов столбцов. Мы будем предполагать, что $\alpha_{i j}-$ элементы поля $G F(p)$. В этом случае $\operatorname{per}\left(A_{m n}\right)$ принимает значения из множества $\{0,1,2, \ldots, p-1\}$.

Нам понадобятся следующие обозначения. Пусть $M=\{1,2, \ldots, m\}-$ конечное множество индексов, соответствующих строкам матрицы $A_{m n}$. Для любого подмножества $I=\left\{i_{1}, i_{2}, \ldots, i_{r}\right\} \subseteq M, 1 \leqslant r \leqslant m$, введем суммы

$$
\xi(I, n)=\sum_{j=1}^{n} \alpha_{i_{1} j} \alpha_{i_{2} j} \ldots \alpha_{i_{r} j}, \quad r=1,2, \ldots, m .
$$

Пусть $\mathscr{K}_{p}=\{K\}-$ множество всех разбиений $K=\left(K_{1}, K_{2}, \ldots, K_{r}\right)$, У которых $k_{i}=\left|K_{i}\right| \leqslant p, i=1, \ldots, r$.

В работе [2] доказана следующая теорема.

Теорема 1. Если элементы матрицы $A_{m n}=\left\|\alpha_{i j}\right\|$ принадлежат полю $G F(p)$, то

$$
\operatorname{per}\left(A_{m n}\right)=\sum_{K \in \mathscr{K}_{p}} c(K) \xi\left(K_{1}, n\right) \xi\left(K_{2}, n\right) \ldots \xi\left(K_{r}, n\right),
$$


где $K=\left(K_{1}, K_{2}, \ldots, K_{r}\right)-$ разбиения множества $M, k_{i}=\left|K_{i}\right|, 1 \leqslant k_{i} \leqslant p$, $i=1,2, \ldots, r, c(K)=(-1)^{m-r}\left(k_{1}-1\right) !\left(k_{2}-1\right) ! \ldots\left(k_{r}-1\right) !$.

Из этой теоремы следует, что для вычисления $\operatorname{per}\left(A_{m n}\right)$ достаточно знать только функции $\xi\left(K_{l}, n\right)$, входящие в сумму в правой части формулы (3). Если матрица $A_{m n}$ случайна, то ее перманент также случаен и его закон распределения определяется суммой в правой части формулы (3). Однако даже в случае, когда все элементы матрицы $A_{m n}$ независимы и равномерно распределены на $G F(p)$, вычислить закон распределения $\operatorname{per}\left(A_{m n}\right)$ затруднительно. Далее мы будем рассматривать последовательность матриц $A_{m n}, A_{m n+1}, A_{m n+2}, \ldots$ с фиксированным числом строк и растущим числом столбцов. Обозначим

$$
\eta_{m}(n)=\operatorname{per}\left(A_{m n}\right)
$$

Тогда формулу (3) можно записать в следующем виде

$$
\eta_{m}(n)=\sum_{K \in \mathscr{K}_{p}} c(K) \xi\left(K_{1}, n\right) \xi\left(K_{2}, n\right) \ldots \xi\left(K_{r}, n\right) .
$$

Далее нам понадобится следующая теорема.

Теорема 2. Если в последовательности матриц $A_{m n}, n=m+1, m+2, \ldots$, столбцы независимы и существует такое $\varepsilon>0$, что для всех $n$ и всех наборов $i_{1}, \ldots, i_{r}$, $1 \leqslant i_{1}<\ldots<i_{r} \leqslant m$, выполняется неравенство

$$
\mathbf{P}\left\{\alpha_{i_{1} n} \alpha_{i_{2} n} \ldots \alpha_{i_{r} n} \neq 0\right\}>\varepsilon,
$$

то все случайные величины $\xi\left(K_{l}, n\right)$ при $n \rightarrow \infty$ сходятся по распределению к предельным случайным величинам $\xi\left(K_{l}\right)$. Все эти предельные величины равномерно распределены на $G F(p)$ и независимы.

В работе [2] доказана аналогичная теорема при более слабых условиях. Иногда вместо $\xi\left(K_{l}\right)$, где $K_{l}=\left\{i_{1}, i_{2}, \ldots, i_{r}\right\} \subseteq M, 1 \leqslant i_{1}<i_{2}<\ldots<i_{r} \leqslant m, 1 \leqslant r \leqslant m$, будем писать $\xi_{K_{l}}$, либо $\xi_{i_{1} i_{2} \ldots i_{r}}$. Из теорем 1 и 2 вытекает следующее утверждение.

Теорема 3. Если выполняются условия теорем 1 и 2, то при $n \rightarrow \infty$ случайная величина $\eta_{m}(n)$ сходится по распределению к случайной величине

$$
\eta_{m}=\sum_{K \in \mathscr{K}_{p}} c(K) \xi\left(K_{1}\right) \xi\left(K_{2}\right) \ldots \xi\left(K_{r}\right)
$$

Из независимости и равномерности распределения случайных величин $\xi\left(K_{1}\right)$, $\xi\left(K_{2}\right), \ldots, \xi\left(K_{r}\right)$ в формуле (5) следует, что там можно убрать множитель $c(K)$. Далее мы будем использовать формулу

$$
\eta_{m}=\sum_{K \in \mathscr{K}_{p}} \xi\left(K_{1}\right) \xi\left(K_{2}\right) \ldots \xi\left(K_{r}\right)
$$

в том смысле, что в этом равенстве совпадают законы распределения правой и левой части. Из формулы (6) следует, что вероятности $\mathbf{P}\left\{\eta_{m}=l\right\}$ при $l=1,2, \ldots, p-1$ принимают одинаковые значения и

$$
\mathbf{P}\left\{\eta_{m}=0\right\}=1-(p-1) \mathbf{P}\left\{\eta_{m}=1\right\} .
$$


В частном случае при $p=3$ формула (6) имеет следующий вид

$$
\begin{aligned}
\eta_{1} & =\xi_{1}, \\
\eta_{2} & =\xi_{1} \xi_{2}+\xi_{12}, \\
\eta_{3} & =\xi_{1} \xi_{2} \xi_{3}+\xi_{1} \xi_{23}+\xi_{2} \xi_{13}+\xi_{3} \xi_{12}+\xi_{123}, \\
\eta_{4}= & \xi_{1} \xi_{2} \xi_{3} \xi_{4}+\xi_{1} \xi_{2} \xi_{34}+\xi_{1} \xi_{3} \xi_{24}+\xi_{1} \xi_{4} \xi_{23}+\xi_{12} \xi_{3} \xi_{4}+\xi_{13} \xi_{2} \xi_{4}+\xi_{14} \xi_{2} \xi_{3} \\
& \quad+\xi_{12} \xi_{34}+\xi_{13} \xi_{24}+\xi_{14} \xi_{23}+\xi_{123} \xi_{4}+\xi_{124} \xi_{3}+\xi_{134} \xi_{2}+\xi_{234} \xi_{1},
\end{aligned}
$$

и так далее.

\section{2. Рекуррентные формулы}

Далее будем использовать обозначение

$$
a_{m}=\mathbf{P}\left\{\eta_{m}=1\right\}
$$

Как было отмечено выше, при любом простом числе $p$ вероятность $a_{m}$ определяет все распределение $\mathbf{P}\left\{\eta_{m}=k\right\}, k=0,1,2, \ldots, p-1$. Для каждого простого числа $p$ введем целые числа

$$
b_{m}(k)=\sum_{l=0}^{k-1}\left(\begin{array}{c}
m-1 \\
l
\end{array}\right), \quad 1 \leqslant k<p<m .
$$

Иначе числа (7) можно вычислять последовательно

$$
b_{m}(k)=b_{m}(k-1)+\left(\begin{array}{c}
m-1 \\
k-1
\end{array}\right), \quad 1 \leqslant k \leqslant m-1, \quad b_{m}(0)=0 .
$$

Теорема 4. Если справедлива формула (6), то при любом $m>p$ имеет место рекуррентная формула

$$
a_{m}=\sum_{k=1}^{p}\left(\gamma^{b_{m}(k-1)}-\gamma^{b_{m}(k)}\right) a_{m-k}, \quad m>p,
$$

где $b_{m}(0)=0, a_{1}=a_{2}=\ldots=a_{p}=\gamma, \gamma=1 / p$.

Доказательство. Для простоты вычислений доказательство проведем при $p=3$. В этом случае выкладки будут короче, а суть доказательства в общем случае аналогична. При $p=3$ формула (9) имеет вид

$$
a_{m}=(1-\gamma) a_{m-1}+\left(\gamma-\gamma^{m}\right) a_{m-2}+\left(\gamma^{m}-\gamma^{\left(\begin{array}{c}
m \\
2
\end{array}\right)+1}\right) a_{m-3}, \quad m>3,
$$

$a_{1}=a_{2}=a_{3}=\gamma, 0=b_{m}(0), m=b_{m}(1),\left(\begin{array}{c}m \\ 2\end{array}\right)+1=b_{m}(2)$. Переобозначим случайные величины $\xi_{m}, \xi_{i m}, 1 \leqslant i \leqslant m-1, \xi_{i j m}, 1 \leqslant i<j \leqslant m-1$, и положим

$$
\theta_{1}=\xi_{m}, \quad \theta_{k}=\xi_{i_{k} m}, \quad 2 \leqslant k \leqslant m-1, \quad \theta_{k}=\xi_{i_{k} j_{k} m}, \quad m \leqslant k \leqslant\left(\begin{array}{c}
m \\
2
\end{array}\right)+1 .
$$


В этих обозначениях события $S_{k}=\left\{\theta_{k}=0\right\}, 1 \leqslant k \leqslant\left(\begin{array}{c}m \\ 2\end{array}\right)+1$, независимы,

$$
\mathbf{P}\left\{S_{k}\right\}=\gamma, \quad \mathbf{P}\left\{\bar{S}_{k}\right\}=1-\gamma, \quad 1 \leqslant k \leqslant\left(\begin{array}{c}
m \\
2
\end{array}\right)+1 .
$$

Введем события

$$
\begin{aligned}
H_{1} & =\bar{S}_{1}, \\
H_{k} & =S_{1} S_{2} \ldots S_{k-1} \bar{S}_{k}, \quad 2 \leqslant k \leqslant\left(\begin{array}{c}
m \\
2
\end{array}\right), \\
H_{\left(\begin{array}{c}
m \\
2
\end{array}\right)+1} & =S_{1} S_{2} \ldots S_{\left(\begin{array}{c}
m \\
2
\end{array}\right)+1} .
\end{aligned}
$$

Далее воспользуемся формулой полной вероятности

$$
\mathbf{P}\left\{\eta_{m}=1\right\}=\sum_{k=1}^{\left(\begin{array}{c}
m \\
2
\end{array}\right)+1} \mathbf{P}\left\{H_{k}\right\} \mathbf{P}\left\{\eta_{m}=1 \mid H_{k}\right\} .
$$

Легко видеть, что

$$
\begin{aligned}
\sum_{k=1}^{\left(\begin{array}{c}
m \\
2
\end{array}\right)+1} \mathbf{P}\left\{H_{k}\right\} & =1, \\
\mathbf{P}\left\{\eta_{m}=1 \mid H_{1}\right\} & =a_{m-1}, \\
\mathbf{P}\left\{\eta_{m}=1 \mid H_{k}\right\} & =a_{m-2}, \quad 2 \leqslant k \leqslant m, \\
\mathbf{P}\left\{\eta_{m}=1 \mid H_{k}\right\} & =a_{m-3}, \quad m<k \leqslant\left(\begin{array}{c}
m \\
2
\end{array}\right), \\
\mathbf{P}\left\{\eta_{m}=1 \mid H_{\left(\begin{array}{c}
m \\
2
\end{array}\right)+1}\right\} & =0 .
\end{aligned}
$$

Отсюда вытекает формула (10). Начальные значения $a_{1}=a_{2}=a_{3}=\gamma$ следуют из формулы (6). Теорема доказана.

Введем обозначения

$$
\Delta a_{m}=a_{m}-a_{m-1}, \quad m=2,3, \ldots
$$

Легко видеть, что $\Delta a_{m}=0$ при $2 \leqslant m \leqslant p$. Очевидны также равенства

$$
a_{m_{2}}=a_{m_{1}}+\sum_{k=m_{1}+1}^{m_{2}} \Delta a_{k}, \quad m_{2}>m_{1},
$$

в частности,

$$
\begin{aligned}
& a_{m}=a_{m-1}+\Delta a_{m}, \\
& a_{m}=a_{1}+\sum_{k=2}^{m} \Delta a_{k}, \\
& a_{m}=a_{p}+\sum_{k=p+1}^{m} \Delta a_{k}, \quad m>p .
\end{aligned}
$$


Далее для вычисления вероятностей $a_{m}$ более удобно вместо формулы (9) использовать некоторую ее модификацию.

Теорема 5. Если выполнены условия теоремь 4, то при $m>$ р справедлива формула

$$
\Delta a_{m}=-\sum_{k=1}^{p-1} \gamma^{b_{m}(k)} \Delta a_{m-k}-\gamma^{b_{m}(p)} a_{m-p},
$$

где $\Delta a_{k}=0$ при $k=2,3, \ldots, p$.

Эквивалентность формул (9) и (12) проверяется элементарно.

\section{3. Вычисление вероятностей $a_{m}$}

При $p=2$ в работе [1] с помощью формулы (6) были вычислены вероятности

$$
a_{2 m-1}=a_{2 m}=\prod_{k=1}^{m}\left(1-\gamma^{2 k-1}\right), \quad \gamma=1 / 2 .
$$

С помощью формулы (12) и равенства

$$
a_{m}=a_{m-1}+\Delta a_{m}
$$

можно последовательно вычислять $a_{m}, m=1,2, \ldots$ Например, при $p=3$ формула (12) имеет вид

$$
\Delta a_{m}=-\gamma \Delta a_{m-1}-\gamma^{m} \Delta a_{m-2}-\gamma^{\left(\begin{array}{c}
m \\
2
\end{array}\right)+1} a_{m-3}, \quad m>3,
$$

где $\gamma=1 / 3, a_{1}=a_{2}=a_{3}=\gamma$. Подставляя $m=4,5,6$ в (12) и используя равенство $a_{m}=a_{m-1}+\Delta a_{m}$, получаем, что

$$
\begin{aligned}
& \Delta a_{4}=-\gamma^{8}, \quad \Delta a_{5}=\gamma^{9}-\gamma^{12}, \quad \Delta a_{6}=-\gamma^{10}+\gamma^{13}+\gamma^{14}-\gamma^{17}, \\
& a_{4}=\gamma-\gamma^{8}, \quad a_{5}=\gamma-\gamma^{8}+\gamma^{9}-\gamma^{12}, \quad a_{6}=\gamma-\gamma^{8}+\gamma^{9}-\gamma^{12}-\gamma^{10} \\
& +\gamma^{13}+\gamma^{14}-\gamma^{17}
\end{aligned}
$$

где $\gamma=1 / 3$. В работе [2] вероятности $a_{5}$ и $a_{6}$ вычислялись с помощью сложной формулы (6) и результат был ошибочным.

\section{Список литературы}

1. Ляпков Л. А., Севастьянов Б. А., Распределение вероятностей перманента случайной булевой матрицы. Дискретная математика (1990) 2, №2, 138-144.

2. Ляпков Л. А., Севастьянов Б. А., Предельное распределение вероятностей перманента случайной матрицы в поле $G F(p)$. Дискретная математика (1996) 8, №2, 3-13.

Статья поступила 25.03.2010. 